Radiologe $2016 \cdot 56: 474-475$

DOI 10.1007/s00117-016-0114-x

Online publiziert:27. Mai 2016

๑) Springer-Verlag Berlin Heidelberg 2016

CrossMark

\author{
M. Uhl' S. Delorme ${ }^{2}$ \\ ${ }^{1}$ Klinik für Diagnostische und Interventionelle Radiologie, Kinderradiologie und Neuroradiologie SJK, RKK- \\ Klinikum Freiburg, Freiburg, Deutschland \\ ${ }^{2}$ Abteilung Radiologie, Deutsches Krebsforschungszentrum, Heidelberg, Deutschland
}

\title{
Knochentumoren
}

\section{Radiologisch-pathologische Korrelation}

Liebe Leserinnen und Leser,

wer erinnert sich nicht voller Grauen an das stumpfe Auswendiglernen der verschiedenen Knochentumoren vor der Facharztprüfung? Wem sank nicht der Mut, als beim Quiz, sei es auf dem Röntgenkongress, dem ECR (European Congress of Radiology) oder dem jährlichen RSNA-Treffen (Radiological Society of North America), die Koryphäe auf dem Podium systematisch die ihm präsentierte Osteolyse differenzialdiagnostisch einkreiste, um dann mit spielerischer Leichtigkeit die richtige Diagnose $\mathrm{zu}$ stellen? In diesem Fall handelte es sich um eine im Übrigen fünfmal in der Literatur beschriebene Entität, im Rahmen irgendeines hereditären Syndroms, bestehend aus einer Kombination mit drei Eigennamen ...

Primäre Knochentumoren sind nicht nur eine diffizile Angelegenheit - sie sind auch selten. So hat, wer nicht gerade in einem orthopädischen Zentrum arbeitet oder eine jener Koryphäen als Lehrer hat, wenig Gelegenheit, ihre Interpretation zu erlernen oder darin sogar Routine zu entwickeln.

Wir könnten ganz pragmatisch damit umgehen und unser Augenmerk darauf lenken, das Relevante vom Nebensächlichen zu trennen. In diesem Falle wäre das beispielsweise den Orthopäden vom Anrühren einer Do-not-touch-Läsion abzuhalten und ferner die Tumoren zu erkennen, die behandelt werden müssen, und alles Weitere den Akademikern zur Diskussion zu überlassen. Nicht jeder hält dies jedoch für ausreichend. Unser Wissensdrang treibt uns zu verstehen, weshalb Tumoren so oder so aussehen.
Ein fataler Fehler bei der Beurteilung von Knochentumoren ist es, sich achselzuckend auf das Urteil des Pathologen zu verlassen. Durch fehlende klinische Informationen handelt es sich bei der Bewertung vieler Befunde um pure Spekulation. Dem Pathologen ergeht es in Bezug auf die radiologischen Aufnahmen wenig anders. Das radiologische Bild ist vielfach eine essenzielle, zusätzliche Information, ohne die auch er zwischen den Entitäten kaum differenzieren kann. Ferner könnte sich deren Relevanz und Prognose teilweise deutlich voneinander unterscheiden. Ein enger Informationsaustausch zwischen Klinikern, Radiologen und Pathologen ist gerade bei Knochentumoren unverzichtbar.

Die klassische Röntgenaufnahme des Knochens hat im Verlauf der Jahre nichts von ihrem Wert eingebüßt und ist weiterhin unersetzlich. Mit den modernen Schnittbildverfahren aber haben sich die Möglichkeiten beträchtlich erweitert, auch jene Teile des Tumors zu erkennen, die anhand der Röntgenaufnahme allenfalls indirekt nachvollzogen werden können - z. B. die nicht kalzifizierenden Weichteilkomponenten, die Ausdehnung im Markraum und vieles mehr. Auch funktionelle Informationen gehören seit jeher zur Diagnostik, waren aber klassischerweise allein der Szintigraphie zu entnehmen, die den lokalen Knochenumsatz wiedergibt. Hinzu kommt jetzt die moderne Magnetresonanztomographie (MRT), die Informationen über die Durchblutung oder die Diffusion im Gewebe liefert. Somit besteht nun die Gelegenheit, erneut die Morphologie der bildgebenden Diagnostik der pathologischen Anatomie gegenüberzustellen. 
Wir sind der Meinung, dass hier, über die bekannten und gelehrten Prinzipien hinaus, ein zusätzlicher Schlüssel zur Diagnose liegt. Knochenläsionen unklarer Genese könnten somit schneller und sicherer identifiziert werden. Aus diesem Grund haben wir diese Abhandlung über Knochentumoren unter das Leitthema „Radiologisch-pathologische Korrelation" gestellt. Dadurch entstehen kleine Strukturänderungen, da die Beiträge nicht so zahlreich, aber zwangsläufig länger sind, da sowohl radiologische als auch pathologische Aspekte dargestellt werden. Thematisch werden osteo- und chondrogene Tumoren, tumorähnliche Knochenveränderungen (Tumor-like lesions) und eine Gruppe von schwer klassifizierbaren, in der Praxis aber wichtigen, Tumoren behandelt. In manchen Passagen dieser Abhandlung haben wir zudem die Gelegenheit, den Dialog zwischen Radiologen und Pathologen $\mathrm{zu}$ verfolgen, und hoffen, dass er viele Nachahmer findet. Als interessiertem Leser wünschen wir Ihnen bei der Lektüre viele Inspirationen, die uns als Herausgeber bei der Durchsicht der Artikel schon vorab gestattet wurden.

Mit kollegialen Grüßen

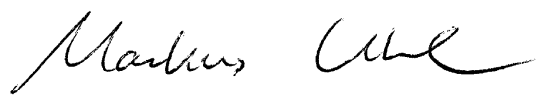

Prof. Dr. Markus Uhl

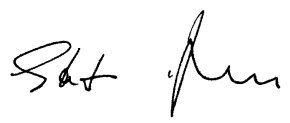

Prof. Dr. Stefan Delorme

\section{Korrespondenzadresse}

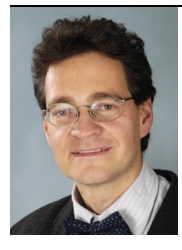

\section{Prof. Dr. M. Uh}

Klinik für Diagnostische und Interventionelle Radiologie, Kinderradiologie und Neuroradiologie SJK, RKKKlinikum Freiburg Sautierstr. 1, 79104 Freiburg, Deutschland markus.uhl@RKK-Klinikum.de

Interessenkonflikt. M. Uhl und S. Delorme geben an, dass kein Interessenkonflikt besteht.

\section{S. Kluge (Hrsg.) \\ Ultraschalldiagnostik der Hand}

Berlin Heidelberg: Springer-Verlag 2015, 1. Auflage, $772 \mathrm{Abb}$., (ISBN 978-3-642-44939-0), über 50 Online-Videos, 149.99 EUR

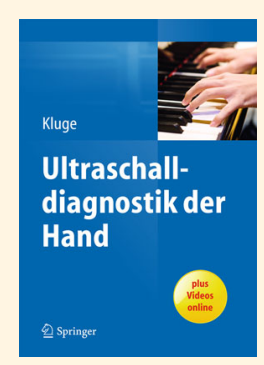

Die Sonografie ist in zahlreichenden Gebieten der Medizin ein etabliertes Untersuchungsverfahren. In der Handchirurgie wird die Sonographie, im Vergleich zu

anderen Gebieten, jedoch noch immer mit großer Zurückhaltung eingesetzt. Das hier vorliegende Buch ist eine hervorragende Anleitung zur sicheren Anwendung der UItraschalldiagnostik an der Hand und kann somit zu einer deutlichen Verbreitung der Sonographie beitragen.

Das Buch gliedert sich zunächst in technische Grundlagen und Untersuchungsgrundlagen und startet dann mit der Sonographie des normalen Gewebes. Hier werden alle wichtigen Gewebearten wie Nerven, Gefäße, Sehnen, Gelenke und Knochen in hervorragender Bebilderung und mit zusätzlichen Videodateien anatomisch/sonographisch suffizient dargestellt. Im Anschluss wird speziell auf Erkrankungen der Hand eingegangen, wie degenerativ veränderte Gewebe, traumatische Läsionen oder neoplastische Veränderungen. Insbesondere die Implantat bedingten Komplikationen werden ebenfalls mit beeindruckendem Bildmaterial und intraoperativen Abbildungen sowie Videos erläutert.

Das Buch verfügt über eine begeisternde Verschmelzung von diagnostischen und klinischen bzw. intraoperativen Befunden. Auf diese Weise werden dem sonographisch unerfahrenen Handchirurgen die Möglichkeiten, Techniken und Vorteile der Ultraschalldiagnostik in den jeweiligen Kapiteln aus allen Blickwinkeln näher gebracht. Das Buch gibt einen großen Überblick über das noch neue Gebiet der Ultraschalldiagnostik der Hand. Für den nicht handchirurgisch tätigen Ultraschalldiagnostiker gibt dieses Werk Einblicke in die handchirurgischen Therapieoptionen und liefert eine sehr gut bebilderte Darstellung der intraoperativen Befunde.

Das hier vorliegende Buch von Kluge "Ultraschalldiagnostik der Hand" sollte in keiner handchirurgischen Bibliothek fehlen und kann allen medizinischen Disziplinen, die sich mit der Diagnostik und Therapie der Hand beschäftigen, uneingeschränkt empfohlen werden.

F. Unglaub (Bad Rappenau) 